\title{
Outcome of adding collagen matrix implant (Ologen) to Trabeculectomy-Trabeculotomy operation with MMC for primary congenital glaucoma
}

\author{
Khulood Muhammad Sayed, MD, PhD, ${ }^{1}$ \\ ${ }^{1}$ The Department of Ophthalmology, Sohag Faculty of medicine, Sohag University, Sohag, \\ Egypt
}

\begin{abstract}
Purpose

To study the value of adding collagen matrix $(\mathrm{CM})$ to the surgical procedure combined trabeculotomy-trabeculectomy with mitomycin C (CTTM) for the treatment of Primary congenital glaucoma (PCG).

\section{Patients and methods}

Fourty eyes were operated upon. The CM was inserted under the scleral and conjunctival flaps. Follow up was for $6-12$ months

\section{Results}

At 6 months; all eyes manifested full success. At 1 year; one eye manifested poor success. There were minimal complications in the form of intraoperative hyphema, vitreous loss and postoperative shallow anterior chamber, immunological reaction and thin avascular cystic blebs.

\section{Conclusion}

This study is novel in using both MMC and CM implant at the same time for augmentation of CTT procedure with highest success ever reported for PCG. Biodegradable CM markedly increased the short and long term surgical success with marked reduction of complications.
\end{abstract}

\section{Introduction}

Congenital glaucoma is a global problem and poses a diagnostic and therapeutic challenge to the ophthalmologist. Detailed evaluation under general anesthesia (GA) is advisable to establish the diagnosis and plan for its management. Medical therapy has a limited role, and surgery remains the primary therapeutic modality. (1)

Trabeculectomy surgery is one of the most common procedures performed in the management of patients with glaucoma since its introduction 40 years ago. (2)

Histological studies have shown the proliferation of subconjunctival fibroblasts, which is believed to play an important role in bleb failure.(3)
Adjunctive antimetabolites, such as 5fluorouracil (5-FU) and mitomycin-C, are commonly used to enhance the success of trabeculectomy. (4)

These agents inhibit fibroblastic proliferation in wound healing, thus

preventing excessive scar tissue formation at the level of the subconjunctival plane. (5)

Although goniotomy or trabeculotomy ab externo is valuable in the management of congenital glaucoma, primary combined trabeculotomytrabeculectomy offers the best hope for success in advanced cases. Trabeculectomy with antifibrotic agents and using glaucoma drainage devices 
has a role in the management of refractory cases. (6)

The Ologen collagen matrix implant is a disc-shaped porcine derived biodegradable collagen matrix that has been developed to prevent excessive scarring after trabeculectomy. It is composed of a three-dimensional porous structure of collagen glycosaminoglycan copolymers. (5) It is designed to prevent episcleral fibrosis and subconjunctival scarring and minimize the random growth of fibroblasts, instead promoting their growth through the pores in the matrix. (7) During trabeculectomy surgery, the implant is placed on top of the sclera flap before the conjunctiva is closed over it. (5) This implant is found to be biodegradable within 90-180 days. (7)

The implant aims to regulate aqueous flow by keeping pressure on top of the sclera flap and by acting as a reservoir as aqueous humour gets absorbed into its porous structure.(8)

After degradation, the implant leaves behind a loose alignment of collagen fibers inside the bleb, which are remarkably similar to normal tissues. (9) In our country and especially in Upper Egypt where the study is carried out, there is a high rate of consanguinity with development of severe forms of congenital glaucoma noted by parents immediately after birth by markedly cloud and large cornea, we believe that this is the most severe and difficult form to manage with high failure rate. The traditional surgery for congenital glaucoma in our department was combined trabeculotomy-trabeculectomy with MMC (CTT-M). Apart from the long term gradual decrease in the success rate of this technique we noticed that some operated eyes developed short term failure as early as 1 week postoperatively with marked corneal edema and high IOP without obstruction of the created fistula as seen on gonioscopy.

This may be because fibrosis in children is more extensive than in adults especially in the early postoperative period. So we though of using a spacer as ologen to ensure the patency of the fistula in the early postoperative period to prevent the short term failure due to active fibroblastic activity in theses babies. Keeping in mind the complications of antimetabolites as well as the complications of glaucoma drainage devices.(1), the idea of using $\mathrm{CM}$ (Ologen) combined with lower dose of antimetabolite was also considered.

So we aim to study the safety, efficacy and the value of adding collagen matrix (CM) Ologen to the procedure (CTTM) for the treatment of PCG . Such study to our knowledge - was not done before.

\section{Patients and methods:}

This study was conducted from February 2015 to October 2016. All children were operated upon after taking informed consent from their parents. All surgeries were performed by a single surgeon.

Inclusion criteria:

All infants with PCG more than 2 months of age

Exclusion criteria:

Children less than 2 months of age, congenital glaucomas associated with another eye anomalies as aniridia or anterior segment dysgenisis and children with less than 6 months follow up period (FUP) were excluded.

The following data was collected : age , gender pre-and postoperative horizontal corneal diameter $(\mathrm{mm})$, intraocular pressure (IOP; $\mathrm{mmHg}$ ), optic disc vertical cup/disc ratio (VC/D) when possible, intraoperative and postoperative complications. 
The data was collected under general anaesthesia . Inhalational sevoflorane (fluoromethyl hexafluoroisopropyl ether) anesthesia was used for all children. The IOP was measured once the eyes became centralized using Goldmann applanation method (Perkin's tonometer, Haag Streit USA and Reliance Medical Products, Mason, OH, USA))

Horizontal corneal diameter (white to white) was measured using calipers. Assessment of the anterior segment and funds examination was carried out when cornea was clear enough to allow this.

In bilateral cases surgery was performed in the fellow eye after 2 week(s)- 1 month according to the condition of the 1 st eye.

Surgical procedure

Surgery was performed by one surgeon (KMS) under general anesthesia. Corneal stay suture was placed using 7-0 0r 8-0 vicryl or virgin silk. A fornixbased conjunctival flap was raised after saline injection to separate the conjunctiva from tenon capsule followed by cutting of tenon capsule as much as possible at the surgical site. A $4 * 4 \mathrm{~mm}$ partial thickness triangular sclera flap was dissected into about $1 \mathrm{~mm}$ of clear cornea. Application of MMC $0.04 \%$ in the conjunctival pockets and sclaeral bed for 1-2 mins. Copious irrigation was done by $40 \mathrm{cc}$ saline. A side port paracentesis was done in the form of a self sealed long tunnel then a radial incision was then carried out across the inner trabeculectomy site (between the junction of surgical limbus and sclera)until the Schlemm's canal was entered; Trabeculotomy was then performed using the internal arm of trabeculotome probes, first to the left then to the right to complete about 100 120 degrees of the circumference. A $2 * 2 \mathrm{~mm}$ inner trabeculectomy block was removed, peripheral iridectomy was performed .The partial thickness scleral flap was sutured using one interrupted suture at apex with 10-0 nylon. The ologen ${ }^{\circledR}$ Collagen Matrix (Aeon Astron Europe B.V.,. Leiden, The Netherlands) was inserted one below and one above the sclera flap. Closure of the conjunctiva was done using mattress sutures (10.0 nylon). (Figure 1)

An eye patch and eye shield were applied. Postoperative treatment included cyclopentolate $1 \%$ five times per day for 2 weeks and dexamethazone $0.1 \%$ every 1 hour for 1 month then 5 times for another month then 2 times per day for another 2 months and moxifloxacin hydrochloride every hour for 5 days then 5 times per day for 1 month. Postoperative treatment was modified according to the condition of the eye and state of the anterior chamber and IOP.

The plan was based on evaluation at four follow up visits under GA at 1 week, 1 , 3 , and 6 months then according to the result of last examination. Conjunctival suture removal was done 1 month after the surgery.

The definition of success was as follows: (1) Full success: IOP less than $15 \mathrm{mmHg}$ without medications; clear cornea.

(2) Satisfactory success: IOP less than $21 \mathrm{mmHg}$ without medications; clear cornea, stable corneal diameter and CDR (3) Poor success: IOP less than 21 $\mathrm{mmHg}$ with medications without progressive cormeal enlargement or progressive cupping

(4) Failure: IOP more than $21 \mathrm{mmHg}$ or less than 21 but progressive cormeal enlargement or progressive cupping

\section{Statistical analysis}

Data was captured using Microsoft Office Excel and analyzed using SPSS 13.0 for windows software. Categorical 
data as percentages $(\%)$ and continuous numerical data were captured as mean and standard deviation (SD). The difference of means was determined using paired t-test. A P value of 0.05 was considered statistically significant.

\section{Results}

A total of 40 eyes of 24 children met the inclusion criteria. There were 23 eyes of males and 17 eyes of females. Twenty were Rt. eyes and 20 were lt. eyes. The preoperative mean age was $7.35 \pm 10.89$ months (range, 2-48 months ). Nineteen $(79 \%)$ of the infants had bilateral disease. (Table 1)

The mean preoperative IOP was $24.25 \pm 8.67 \mathrm{mmHg}$ (range 14-40). Intraoperative complications included: premature entrance of the anterior chamber (AC) (2 eyes) hyphema (3 eyes) and vitreous pocket herniation in one eye.

In the 2 eyes with premature entrance of the $\mathrm{AC}$, one eye was treated by completion of surgery without MMC application. The 2 nd eye was treated by tight closure of the sclera flap and changing the site of surgery.

The 3 eyes with hyphema did not need intervention and it was resolved within the 1 st postoperative week.

The eye with vitreous pocket herniation was managed with quick tight closure of the sclera flap and positioning of the ologen CM implant under the sclera flap which pushed and allowed for repositioning of the vitreous pocket.

Postoperative results:

Mean postoperative IOP was $0.85,6.5,7.25 .7 .15$ and $6.23 \mathrm{mmHg}$ at 1 week. 1,3,6 months and 1postoperative year respectively (Figure 2)

IOP at the 1st postoperative week was significantly lower than the IOP at the 1st postoperative month $(\mathrm{P}=0.00)$ then no significant difference was detected between each follow up visit and the preceding one. (Table 3)

There was a statistically significant reduction in IOP at all postoperative periods of follow up $(\mathrm{P}=0.00)$

Corneal clarity

All the eyes had some degree of corneal edema preoperatively, with subsequent improvement resulting in clear cornea in majority of the eyes postoperatively (Figure 3 ).

Postoperative complications: (Table 4)

1.Shallow anterior chamber were noticed in 5 eyes $(10 \%)$ all were improving with gradual deepening at different periods within the 2 postoperative weeks during this period we use only one drop of steroid per day instead on one drop hourly then gradually increase the dose by one or 2 drops as the anterior chamber deepens till reach the usual dose. Non of the eyes entered in postoperative choroidal detachment or hemorrhage; red reflex was bright even in extremely shallow anterior chamber. Ultrasound was not done routinely for eyes with shallow AC for fear of infection and pressure by the U/S probe on a markedly hypotonous globe except if red reflex color is suspicious which was not happened in this study

2.Immunological reaction to Ollogen matrix implant was noticed in 2 children $(8.3 \%)$, the $1 \mathrm{st}$ is $2 \mathrm{~ms}$ old baby (case no. 1 in the series) immune reaction was happened in the form of upper lid edema in the 1st postoperative day which was successfully treated by adding systemic steroid and antihistamincs for one week, edema did not recur again.

The other baby was $4 \mathrm{~ms}$ old baby (case no.23 in the series) complained of postoperative marked bilateral blepharospam, lacrimation and buffy eyelids. At 1st we thought it to be 
from the prominent conjunctival stitches (nylon 10-0) we add tear substitutes to the usual postoperative medications till we removed the conjunctival stitches after $1 \mathrm{~m}$ from surgery in both eyes, on suture removal and examination under general anasthesia with the handheld slit-lamp and corneal staining with sterile fluorescein stain, the eye was so quiet, no corneal staining, no infection and IOP was controlled (5 and $6 \mathrm{mmHg}$ in Rt. and Lt. eyes respectively) the symptoms continued after stitch removal and increased on withdrawal of postoperative steroids eye drops so we consider it to be an immunological reaction to ollogen and manage him with systemic weak antihistaminc now he is doing well after 9 m.s of surgery. (Figure 4)

3.Thin avascular cystic bleb

Were noticed in 8 eyes (20\%) of 5 children starting to appeared at different periods from the 1st to 5th postoperative months none of them was accompanied by aqueous sweating (all were -ve Seidle test) (figure 5 ). All eyes were exposed to MMC $0.04 \%$ for 2 min.s, so we shifted to $1 \mathrm{~min}$. exposure during the same study in the last 20 eyes of the series. We managed these cases with potent tear substitute to avoid friction of the bleb with the upper eyelid movements and tobramycin or gentamycin eye drops and ointment both as a prophylaxis against bleb infection and to induce scarring action as both irritate the surface of the bleb, so we believe that this may stimulate epithelial cells to proliferate. Autologus serum was used in 2 eyes with markedly elevated thin bleb. These actions were taken to save the bleb surface before becoming leaky.

4. cataract formation:

2 eyes (5\%) developed faint insignificant anterior subcapsular cataract both suffered from postoperative shallow anterior chamber for more than 1 week.

No serious complications as postoperative choroidal detachment, or suprachoroidal hemorrhage were noted .

Surgical success:

At the 6th postop.month full success was achieved in $100 \%$ of eyes. Among 17 eyes completed 1 year follow up full success was achieved in all eyes except one eye which achieved poor success IOP was $16 \mathrm{mmHg}$ with medication $(5.9 \%)$.

We did not see any eye with early postoperative failure (within the 1 st postoperative month)

\section{Tables:}

Table 1. Patients demographic data

\begin{tabular}{|l|l|l|l|}
\hline Item & No. & No. & total \\
\hline Sex & 23 males & 17 females & 40 eyes \\
\hline laterality & 19 bilateral disease & 5 unilateral & 24 child \\
\hline Eye & $20 \mathrm{OD}$ & $20 \mathrm{OS}$ & 40 eyes \\
\hline & & & \\
\hline & Mean \pm SD & Range & \\
\hline Mean age & $7.35 \pm 10.89 \mathrm{months}$ & $2-48 \mathrm{months}$ & \\
\hline $\begin{array}{l}\text { mean preoperative } \\
\text { IOP }\end{array}$ & $24.25 \pm 8.67 \mathrm{mmHg}$ & $14-40 \mathrm{mmHg}$ & \\
\hline
\end{tabular}

Table 2 : Intraoperative complications

\begin{tabular}{|l|l|l|}
\hline Complication & No. of eyes & Percentage \\
\hline $\begin{array}{l}\text { Premature entrance of the anterior } \\
\text { chamber }\end{array}$ & 2 eyes & $5 \%$ \\
\hline Hyphema & 3 eyes & $7.5 \%$ \\
\hline Vitreous pocket herniation & one eye. & $2.5 \%$ \\
\hline
\end{tabular}


Table 3 : Mean IOP difference between follow up periods

\begin{tabular}{|l|l|l|}
\hline Period of follow up & IOP & P-value \\
\hline 1week-1month & $0.85-6.5 \mathrm{mmHg}$ & $0.00^{*}$ \\
\hline 1month-3months & $6.5-7.25 \mathrm{mmHg}$ & 0.135 \\
\hline 3months-6months & $7.25-7.15 \mathrm{mmHg}$ & 0.779 \\
\hline 6months-1year & $7.15-6.23$ & 0.646 \\
\hline *statistically significant &
\end{tabular}

Table 4 : Postoperative complications

\begin{tabular}{|l|l|l|}
\hline Complication & No. of eyes & Percentage \\
\hline Shallow anterior chamber & 5 eyes & $10 \%$ \\
\hline Immunological reaction & 2 children & $8.3 \%$ \\
\hline Thin avascular cystic bleb & 8 eyes & $20 \%$ \\
\hline Cataract formation & 2 eyes & $5 \%$ \\
\hline
\end{tabular}

\section{Figures:}

\section{Figure 1.}

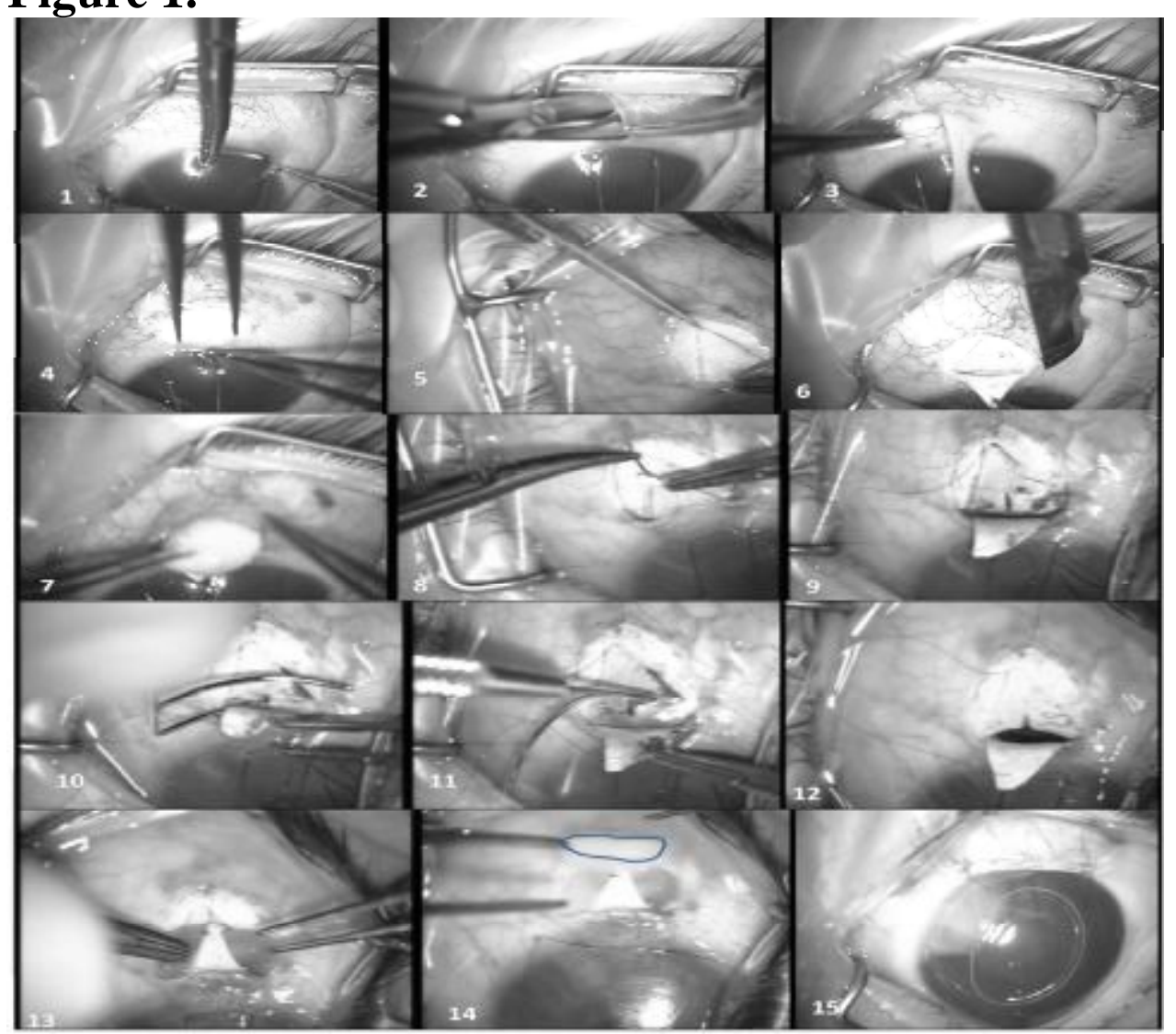

Fig. 1. Surgical steps.

1. Corneal traction suture. 2. Conjunctival dissection. 3. Tenon cutting. 4,5,6. Scleral flap creation. 7. MMC application. 8. Preplaced suture. 9. Deroofing of Schlemm's canal. 10,11. Trabeculotomy of the Lt. and Rt. Sides. 12. Trabeculectomy and peripheral iridectomy. 13.OM under the sclera flap. 14. Another OM over the sclera flap. 15. Closure of the conjunctiva with mattress sutures 
Figure 2.

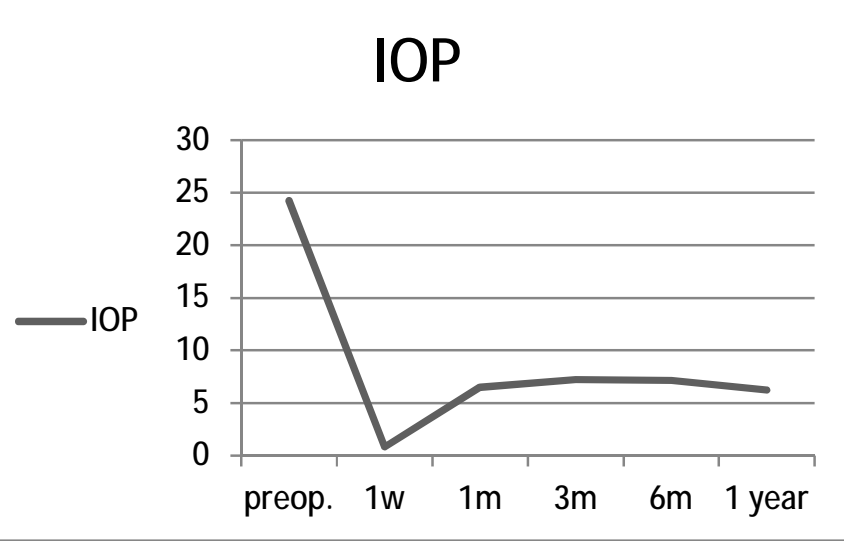

Fig. 2. Postoperative mean IOP within the different follow up periods 


\section{Figure}

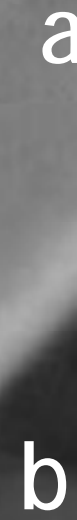

a

b
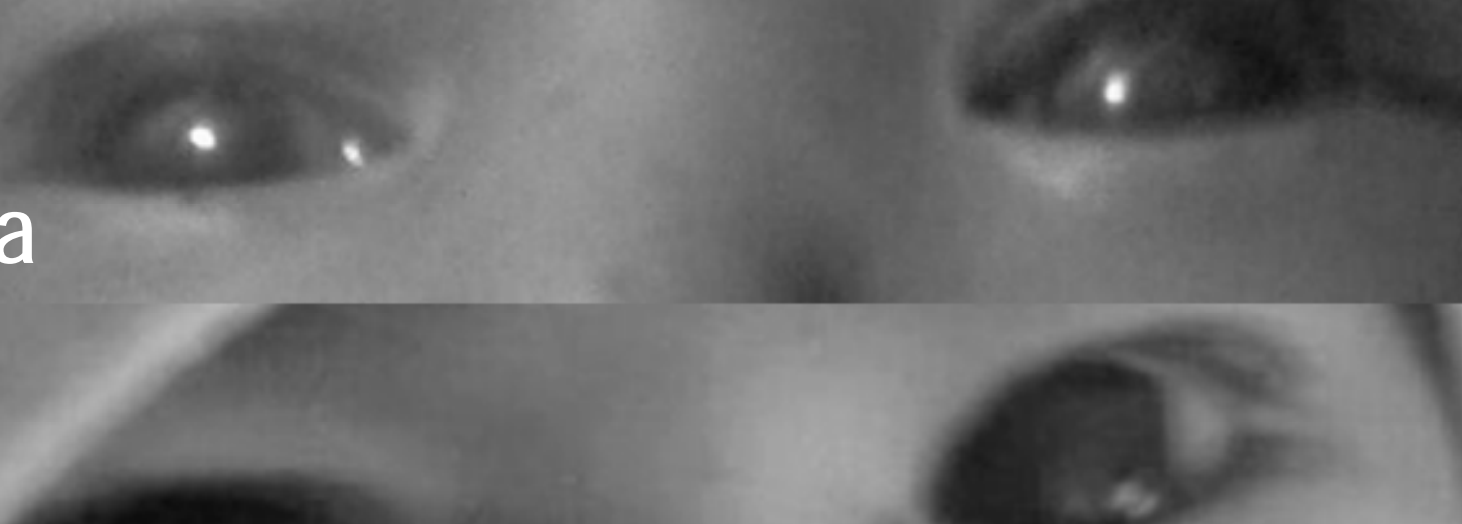

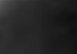

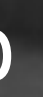

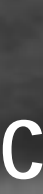

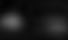

$\theta$

d

Fig. 3. Difference between preoperative and postoperative corneal clarity (case no. 13)

a. Preoperative photo with bilateral cloudy cornea.

b. 1 month postoperatively with partial clearance of the cornea . c. 3 months postoperatively. $d$. represent the same child photos 10 months postoperatively for both eyes with totally clear cornea and spectacle correction. 


\section{Figure 4}

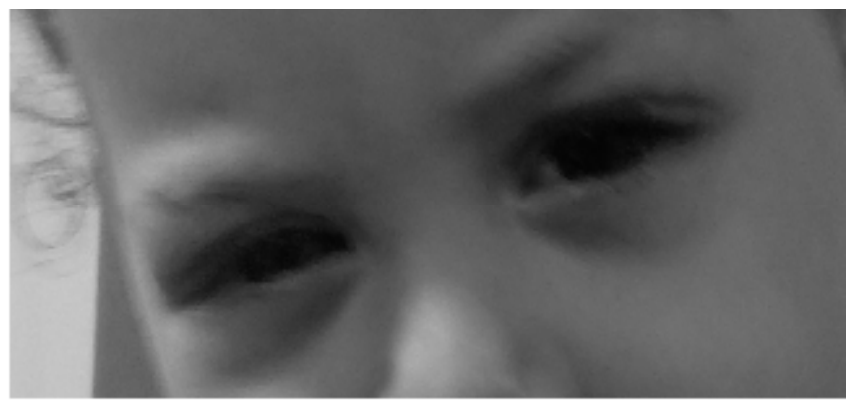

Fig. 4. Immunological reaction (case no. 23 in the study)

Immunological reaction during the course of treatment with antihistamincs surgery).

(8 months after

\section{Figure 5}

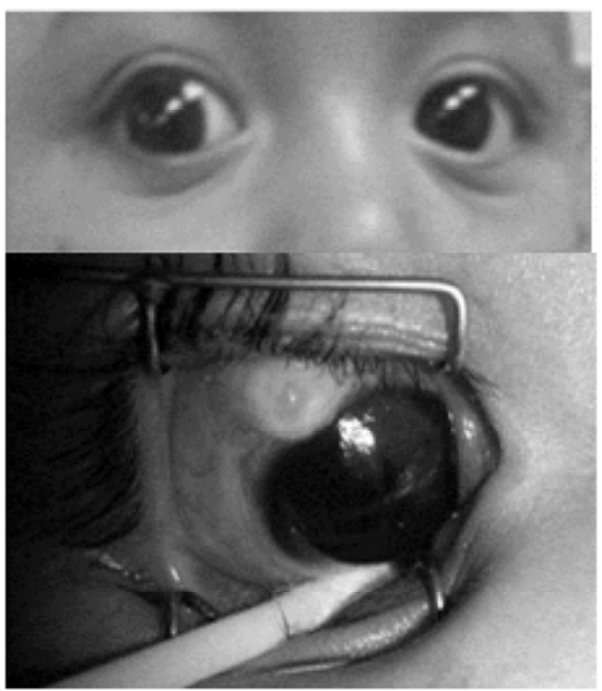

Fig. 5. Thin avascular cystic bleb (case no. 4 in the study)

A 4 months old girl developed thin avascular cystic bleb at the $5^{\text {th }}$ postoperative month.

\section{Discussion}

To our knowledge; this study is novel in using both MMC and ologen implant at the same time for augmentation of CTT procedure for this age group .

Trabeculotomy, trabeculectomy, and CTT are the most frequent surgical treatments for

PCG, and their surgical outcomes have been intensively studied. (10)

Children or young adults undergoing filtering surgery don't enjoy the same success as do those of older age group. The barriers to success of filtering surgery in children include thick tenon's capsule, rapid wound healing response, lower sclera rigidity and large buphthalmic eye with thin sclera. (11) The prognosis of the PCG is affected by various factors such as time of presentation, severity and the time of surgery. The earlier in life the disease occurs, the worse the prognosis and the 
more severe the case, the higher is the failure rate. (12) In upper Egypt where this study is curried out; consanguinity is high and the disease presentation is as early as at the time of birth which is the most severe form with marked corneal edema and scarring. So we aimed to increase the success rate of the traditionally used CTTM procedure by adding OM implant especially when we noted 2 eyes suffered from early failure (as early as 1 week after CTTM surgery). There was no block of the site of the fistula as revealed by gonioscope nor postoperative inflammation. Histological studies have shown that proliferation of subconjunctival fibroblasts, which is believed to play an important role in bleb failure, occurs during the third to fifth postoperative day. (3) which can explain this early failure with CTTM.

In this study, CM (ologen) was used in conjunction with CTTM as a surgical treatment of 40 eyes with primary congenital glaucoma. The follow-up was continued for 6 months to 1 year. All cases were completely successful at 6 months follow up and $95 \%$ complete success after 1 year (1 eye only successful with medication) with the absence of significant or visionthreatening complications.

Previous studies conducted on similar patient populations reported lower success rate, such as Elder \& Mandal et al $(13,14)$ who achieved a success rate of $93.5 \%$ in Israel, $94.4 \%$ in India with combined trabeculotomytrabeculectomy without mitomycin $\mathrm{C}$ for both studies respectively. $75 \%$ success was reported at the end of 6 months follow up of CTT with MMC. (11) A German study reported trabeculotomy success rates of $92.6 \%$ after 1 year of follow-up. (15) In Turkey in Yalvac et al
(16) reported that the success rates of trabeculotomy after 12 months were $92 \%$.

This study reported a full success rate of $100 \%$ after 6 months and $94.12 \%$ after 1 year which is higher than ever seen in previous reports

This discrepancy in success rates may be -to a large extend- attributable to the combination of MMC and $\mathrm{OM}$ in this study. We thought of using a spacer as ologen to maintain the subconjunctival and subscleral spaces at the early postoperative period . the $2 \mathrm{nd}$ benefit is to decrease the intraoperative exposure time to MMC from 2 min.s to 1 min. so reducing $\mathrm{MMC}$ related complications. The 3rd benefit of combining MMC and ologen is that MMC inhibits the proliferation of the remaining tenon's capsule into the pores of ologen which may block it so leaving it patent for long time before degradation thus helping in both early and late success.

Regarding complications seen in this study they are much more less than previous reports. (17) study evaluating CTT with MMC reported subconjuctival hemorrhage in $16 \%$ of eyes, false passage of the trabeculotome probe intraoperatively resulting in detached iris root $(5 \%)$ both were not seen in this study. No sight threatening complications were seen. In contrast sight threatening postoperative complication of seclusion pupillae was reported . (17)

Papaconstantinou et al (18) reported Positive Seidel test in $10 \%$, encapsulated bleb $10 \%$ endophthalmitis $5 \%$ when evaluating ologen with trabeculectomy. these were also not seen in this study

The incidence of Avascular, thin, sharply demarcated blebs were reported 
to range from $33.3 \%-66.67 \%$ with MMC of 0.02 and $0.04 \%$. (19)

This complication occurred in $20 \%$ (8 eyes ) in our study.Eyes suffered from this complication were exposed intraoperatively to $2 \mathrm{~min}$ MMC $0.04 \%$. Although the incidence of this complication is less than previous reports we shifted all subsequent surgeries to $1 \mathrm{~min}$ exposure to MMC $0.04 \%$ and we did not notice any avascular thin blebs within the subsequent 20 eyes with 1 min.during the follow up period.

Although reductions in the cup-disc ratio and corneal diameter were seen in successful surgeries reported by Zhang et al (20) in a study done on Chinese population both parameters were not included in our study as most eyes manifested various degrees of corneal edema till corneal scarring with difficult preoperative fundus visualization. TCD was not measured in all eyes so both were excluded. Other studies reported stabilization of TCD . (21)

Shallow anterior chamber occurred in $10 \%$ in our series, none of them required reformation. We believed that postoperative shallow AC and early hypotony is not a big issue when using ologen as when over filtration occurs , ologen absorbs aqueous, becomes larger in size leading to more tamponading effect at the site of trabeculectomy securing it and preventing marked hypotony and its complications such as choroidal detachment and hypotonous maculopathy. (11) reported choroidal detachment of $3.12 \%$ which is not seen in our study.

A limitation of this study is the relatively small number of patients especially those who completed 1 year follow up. Larger patient series and longer follow up periods are needed to get stronger results. Actually at this moment we completed the same surgery for 80 eyes but this study included the initial results of only 40 eyes which completed 6-12 month follow up. We will follow the whole series and the new results will include larger number of operated eyes with longer follow up.

In conclusion; although the use of CTTM is a useful procedure for the management of PCG. The addition of ologen to the surgical procedure markedly increases the short and long term success, decreases the needed dose of the antimetabolites and hence its related complications together with marked reduction of short and long term complications and low learning curve. The author recommends adding ologen to CTT-M surgery for PCG and thought that this procedure would has a rule in eyes with resistant glaucomas as it may be a safer and effective alternative to glaucoma drainage devices. Longer term follow up with larger series is needed to confirm this outcome. These initial results may encourage us to use this procedure for resistant types of paediatric glaucoma.

\section{References}

1. Hamdi MM . Trabeculectomy assisted by collagen matrix implant (Ologen) in primary congenital glaucoma. J Egypt Ophthalmol Soc. 2013; 106:188-193.

2. Cairns JE .Trabeculectomy. Preliminary report of a new method. Am J Ophthalmol. 1968; 66:673-679.

3. Pescosolido N, Scarsella G \& Risuleo G. An in vitro model for posttrabeculectomy: evaluation of drugs differently controlling cell proliferation. Acta Ophthalmol Scand Suppl. 2002;236: 259. 
4. Cillino S, Zeppa L, Di Pace F, Casuccio A, Morreale D, Bocchetta F \& Lodato G. E-PTFE (Gore-Tex) implant with or without low dosage mitomycin-C as an adjuvant in penetrating glaucoma surgery: 2 year randomized clinical trial. Acta Ophthalmol. 2008; 86: 314-321.

5. Boey PY, Narayanaswamy A, Zheng C, Perera SA, Htoon HM, Tun TA, Seah SK, Wong TT, Aung T. Imaging of blebs after phacotrabeculectomy with Ologen collagen matrix implants $\mathrm{Br} \quad$ J Ophthalmol. 2011; 95:340-344.

6. Mandal AK, Chakrabarti D. Update on congenital glaucoma. Indian $\mathbf{J}$ Ophthalmol. 2011; 59 Suppl:S148S157.

7. Sarkisian SR. A replacement for antimetabolites? Ologen is a new product that modulates wound healing in glaucoma surgery. Glaucoma Today. 2010; 8:22-24.

8. Hsu WC, Ritch R, Krupin $\mathrm{T}$, et al. Tissue bioengineering for surgical bleb defects: an animal study. Graefes Arch Clin Exp Ophthalmol. 2008;246:709-717.

9. Ritch R. Using bioengineered collagen matrix during trabeculectomy: biodegradable polymer implants show promise for improving the functioning of filtering blebs. Glaucoma Today. 2007;1:1415.

10. Mandal AK, Gothwal VK, Bagga H, Nutheti R, Mansoori T. Outcome of surgery on infants younger than 1 month with congenital glaucoma. Ophthalmology. 2003; 110:19091915.
11. Reddy PK, Dada $T$, Sihota $R$, Panda A, Khokkar SK; Gupta, V. Comparative Evaluation of Trabeculotomy- Trabeculectomy with Mitomycin C vs Trabeculectomy, with Mitomycin C for Primary Congenital Glaucoma Journal of Current Glaucoma Practice.2011; 5: 15-19.

12. Christopher J, Dickens H, Hoskins D. Epidemiology and pathophysiology of congenital glaucoma. The Glaucomas. 1996; 2:729-738.

13. Elder MJ. Combined trabeculotomy-trabeculectomy

compared with primary trabeculectomy for congenital glaucoma. Br J Ophthalmol. 1994; 78: 745-748.

14. Mandal AK, Naduvilath TJ, Jayagandan A. Surgical results of combined trabeculotomytrabeculectomy for developmental glaucoma. Ophthalmology. 1998; 105: 974-982.

15. Schwenn O, Pfeiffer N, Grehn F. Trabeculotomy in congenital glaucoma. Graefes Arch Clin Exp Ophthalmol. 2000; 238:207-213.

16. Yalvac IS, Satana B, Suveren A, Eksioglu U, Duman S. Success of trabeculotomy in patients with congenital glaucoma operated on within 3 months of birth. Eye. 2007;21:459-464.

17. Essuman VA, Braimah IZ, Ndanu TA and Ntim-Amponsa CT. Combined trabeculotomy and trabeculectomy: outcome for primary Congenital glaucoma in a West African population Eye. 2007; 25: 77-83. 
18. Papaconstantinou

D, Georgalas

I, Karmiris

E, Diagourtas

A, Koutsandrea C, Ladas I, Apostolopoulos M, Georgopoulos $\mathrm{G}$

Trabeculectomy with OloGen versus trabeculectomy for the treatment of glaucoma:

a pilot study. Acta Ophthalmol. 2010; 88:80-85.

19. Agarwal HC, Sood NN, Sihota R, Sanga L, Honavar SG. Mitomycin-
$\mathrm{C}$ in Congenital Glaucoma Ophthalmic Surgery, Lasers and Imaging Retina. 1997; 12: 979-985.

20. Zhang $X$, Du S, Fan Q, Peng S, Yu $\mathrm{M}, \mathrm{Ge} J$. Long-term surgical outcomes of primary congenital glaucoma in China. Clinics. 2009; 64:543-551.

21. Bayoumi NHL. Deep sclerectomy in pediatric glaucoma filtering surgery Eye. 2012; 26:1548-1553 . 